\title{
LA PROTECCIÓN DEL AMBIENTE COMO LÍMITE AL DERECHO DE PROPIEDAD EN ITALIA
}

\author{
Flavio Tovani \\ UNIVERSITÉ DE TOULON
}

\section{Resumen}

Con el fin de proteger el ambiente se pueden establecer varias limitaciones específicas a la propiedad privada. El principio constitucional de la "función social" de la propiedad permite definir un límite de carácter general a las posibilidades de uso del bien por el propietario y, en particular, a la posibilidad de que el mismo haga inmisiones industriales en el fundo del vecino. Este artículo pretende brindar una hipótesis sobre el uso de la propiedad para asegurar la protección del ambiente, todo esto en el marco del Código Civil italiano, en especial a lo que hace referencia al régimen de uso de suelo y la Constitución republicana.

Palabras clave: ambiente, propiedad privada, Derecho Civil, ley.

El autor: abogado, doctor en Persona e tutele giuridiche, de la Scuola Superiore Sant'Anna de Pisa (Italia). Docente e investigador en la Universidad de Toulon (Francia). Dirección postal: Via Lamarmora 177-18038 Sanremo (IM) (Italia).Correo electrónico: flaviotovani@tiscali.it

Recibido: 10 de octubre de 2013; evaluado: 13 de noviembre de 2013; aceptado: 27 de mayo de 2014. 


\title{
ENVIRONMENTAL PROTECTION AS A LIMIT TO PROPERTY RIGHTS IN ITALY
}

\author{
Flavio Tovani \\ UNIVERSITÉ DE TOULON
}

\begin{abstract}
In the attempt of protecting the environment, a number of specific limits to private property could be established. The constitutional principle of the "social function" of property enables the setting of a general limit to the possibilities of use the owner has over the asset, especially to the possibility the owner has of performing industrial immissions into the neighbor's property. The purpose of this paper is to provide a hipothesis about the use of property to assure environmental protection. Everything is set in the context of the Italian civil code, chiefly what makes reference to real estate law and the Constitution of the Roman Republic.
\end{abstract}

Keywords: Environment, Private Property, Civil Law, Law

About the author: Lawyer. Doctor in "Persona e tutele giuridiche" from the Scuola Superiore Sant'Anna de Pisa (Italy). Professor and researcher at the University of Toulon (France). Address: Via Lamarmora 177 - 18038 Sanremo (IM) - Italy. E-mail: flaviotovani@tiscali.it

Received: October 10, 2013; reviewed: November 13, 2013; accepted: May 27, 2014. 


\section{Introducción}

En el Código Civil italiano se destaca un equilibrio de los derechos individuales con el interés público, sobre todo en el régimen del derecho de propiedad. ${ }^{1}$ En la Constitución republicana, el Artículo $41^{2}$ trata, de manera innovadora en relación con los códigos y las Constituciones de los Estados liberales, sobre la "función social" de la propiedad. ${ }^{3}$

En el contexto actual, los mencionados datos normativos permiten una interpretación de la noción de la propiedad como límite a los poderes de los propietarios, con el fin de facilitar el acceso de todos a los "bienes comunes", incluido el ambiente. De otra parte, en Europa se ha ido afirmando la idea de que es posible tutelar el derecho (fundamental y común) al ambiente ${ }^{4}$ sin poner barreras irrazonables al derecho (individual) de propiedad. ${ }^{5}$

No solo nos referimos a varios deberes específicos del propietario para proteger el ambiente, sino también a la disciplina de las inmisiones de tipo industrial consagradas en el Artículo 844, numeral 2 del Código Civil.

En la mente de los redactores del Código Civil, la disciplina de las inmisiones era menos estricta que en la disciplina de las inmisiones en general, ${ }^{6}$ pues contempló el deber del vecino de sufrir inmisiones superiores a la "tolerabilidad normal". Ahora más que nunca se deben tener en cuenta las exigencias ambientales que constituyen un límite tanto para la utilización del predio, por la imposición de varios deberes de índole administrativa, como para la posibilidad de imponer las inmisiones al vecino. ${ }^{7}$

Esto no excluye que la disciplina de las inmisiones en general, así como las reglas de los planes reguladores y otras reglas específicas en torno a la propiedad,

\footnotetext{
Salvatore Pugliatti, La proprietà nel nuovo diritto (Milano: Giuffrè, 1954), 2.

2 "La propiedad es pública o privada. Los bienes económicos pertenecen al Estado, a entidades o personas. La propiedad privada está reconocida y garantizada por la ley, que establece las modalidades de adquisición y de goce y los límites con el fin de asegurar su función social y de hacerla accesible a todos.

La propiedad privada puede ser expropiada, en los casos previstos por la ley, con el pago de la indemnización, por razones de interés general.

La ley establecerá las normas y los límites de la sucesión legítima y testamentaria y los derechos de sucesión del Estado".

3 Paolo Caretti y Ugo de Siervo, Istituzioni di diritto pubblico. 7a ed. (Turín: Giappichelli, 2004), 474.

4 Filippo Gargallo di Castel Lentini, "Lambiente come diritto fondamentale dell'uomo", http://www.dirittoambiente.net/file/vari_articoli_170.pdf (acceso febrero 4, 2012).

5 Sobre esta temática puede verse, entre otros, Stefano Rodota, Il diritto di avere diritti (Roma: Laterza, 2012), 105-138.

6 Andrea Torrente y Piero Schlesinger, Manuale di diritto privato. 10a ed. (Milán: Giuffré, 1978), 314-315.

7 En la doctrina se evidencia la posibilidad de utilizar la disciplina de las inmisiones para la protección de la salubridad del ambiente. Puede verse Massimiliano di Pirro, Istituzione di diritto privato (diritto civile). $7 \mathrm{a}$ ed. (Nápoles: Simone, 2001), 354.
} 
instituidas en el Código Civil, pudiera establecer límites a la propiedad que vayan en la misma dirección.

\section{Algunas limitaciones específicas: los deberes hidrogeológicos y los deberes en las áreas protegidas}

Al tratar de las limitaciones a la propiedad, examinaremos primero los deberes hidrogeológicos, cuya función es importante en la protección del territorio y que ahora, en Italia, es un tema muy débil, debido a una cementación muy fuerte, al abandono de tierras agrícolas y los cambios climáticos. En efecto, los eventos meteorológicos extremos son siempre más frecuentes y la tierra, cubierta de cemento, no absorbe el agua y no obstaculiza aluviones o inundaciones.

Además, como muchas veces sucede, el Código Civil (junto con algunas leyes especiales $)^{8}$ puede anticiparse y, no obstante haber sido redactado en una época en la que las preocupaciones ambientales casi no existían, prevé la posibilidad de imponer estos deberes sobre terrenos, para evitar que pierdan estabilidad o turben el régimen de las aguas con un daño público: se trata de un claro ejemplo de la limitación de la propiedad por el interés público, sin previsión de una indemnización para los propietarios como en los casos de expropiación. ${ }^{9}$

Otra limitación que no es una expropiación sin derecho a la indemnización ${ }^{10}$ consiste en la que viene de la inclusión de un bien de propiedad privada en el área de un parque natural, que puede señalar limitaciones muy fuertes a los derechos de goce y disposición de la cosa por el propietario, quien tiene que conducirse en una dirección eco-compatible.

La Ley 394 de 1991 dispone por primera vez una disciplina orgánica de los diversos deberes en torno a la propiedad. ${ }^{11}$ El Artículo 12 de dicho texto legal ${ }^{12}$ distingue

8 Mauro Renna, "Vincoli alla proprietà e diritto dell'ambiente", http://www.astrid-online.it/Gli-osserv/ llpp/M_Renna-Vincoli-alla-propriet--08_09_06.pdf (acceso febrero 6, 2014).

$9 \quad$ Francesco de Martino, "Della proprietà". 6a ed. (Bolonia: Zanichelli, 1976), 254. En cuanto a las minas, se habla de un interés público sobre su uso, que sucumbe ante un interés público más importante, es decir, la estabilidad hidrogeológica del terreno. Marco Sertorio, "Attività estrattiva e tutela ambientale" http://www. assomineraria.org/news/attach/copy_0_sertorio_attivit_estrattiva_e_tutela_ambientale.pdf (acceso febrero 10, 2014)

10 Maria Antonia Ciocia, Aree protette e diritto di proprietà: vincoli urbanistici ed uso del territorio. (Padua: Cedam, 1999), 42

11 Ciocia, Aree protette e diritto di proprietà, 102. Además, puede verse Carlo Baseggio, "La disciplina giuridica delle aree naturali protette, tra tutela dell'ambiente e governo del territorio", http://amsdottorato.cib.unibo. it/121/1/Tesi_Baseggio_Carlo.pdf (acceso agosto 1, 2012).

12 "1. La protección de los valores naturales y del medio ambiente confiado al ente parque se persigue a través del instrumento del plan para el parque, en lo sucesivo, 'plan, que debe, en particular, regular el contenido siguiente: 


\section{entre las "reservas integrales", "reservas generales orientadas", "áreas de protección" y "áreas de promoción económica y social". En las primeras no es posible ninguna}

a) la organización general del territorio y su articulación en las zonas o partes caracterizadas por las diferentes formas de uso, goce y protección;

b) las restricciones, los destinos de uso público o privado y las normas de aplicación en relación con las distintas áreas o partes del plan;

c) Los sistemas de acceso vehicular y peatonal, con especial atención a los caminos, el acceso y las facilidades para personas con discapacidad, los discapacitados y los ancianos;

d) Los sistemas, equipos y servicios para la gestión y la función social del parque, museos y visitas, oficinas de información, áreas para acampar, agroturismo;

e) directrices y criterios para el trabajo sobre la flora, la fauna y el medio ambiente en general.

2. El plan divide el territorio de acuerdo con los diferentes grados de protección, que debe incluir: a) las reservas naturales donde el entorno natural se conserva en su integridad, b) las reservas generales orientadas, en las que se prohíbe la construcción de nuevas obras de construcción, ampliar los edificios existentes, llevar a cabo las obras de transformación del territorio. Sin embargo, se permitirán los usos tradicionales de producción, la construcción de infraestructuras estrictamente necesarias y las acciones para la gestión de los recursos naturales en el cuidado del parque. Se permiten también las obras de mantenimiento de las obras existentes en las letras a) y b) del párrafo primero del artículo 31 de la Ley 457 de 5 de agosto 1978, c) las zonas de protección en la que, de acuerdo con la finalidad institucional y de acuerdo con los criterios generales establecidos por el parque puede continuar, de acuerdo con los usos tradicionales o con los métodos de la agricultura orgánica, las actividades agro-silvo-pastorales y la pesca y la recolección de productos naturales, y también alienta a la producción de calidad artesanal. Se admite la intervención autorizada conforme a las letras a) , b) y c) del párrafo primero del artículo 31 de la Ley 457 de 1978, sujeto al cumplimiento de las normas del plan sobre el uso previsto, y d) las áreas promoción económica y social, que son parte de un mismo ecosistema, más ampliamente modificadas por los procesos de asentamientos humanos, en los que se permiten actividades compatibles con la finalidad institucional del parque destinadas a mejorar la vida socio-cultural de las comunidades locales y el mejor disfrute del parque por parte de los visitantes.

3. El plan es preparado por el parque dentro de los seis meses de su creación, sobre la base de los principios y los objetivos de la presente ley y adoptada por la región dentro de los cuatro meses siguientes, en consulta con las autoridades locales.

4. El plan adoptado se deposita durante cuarenta días en las oficinas de los municipios, las comunidades de montaña y las regiones en cuestión, y cualquier persona puede examinar y obtener copias de los mismos. Dentro de los cuarenta días siguientes cualquier persona puede presentar observaciones por escrito, en el que la Autoridad del Parque emitirá su dictamen en un plazo de treinta días. Dentro de ciento veinte días a partir de la recepción de este dictamen, la región se pronuncie sobre las observaciones formuladas y, de acuerdo con la Autoridad del Parque en los ámbitos mencionados en las letras a), b) y c) del apartado 2 y en el acuerdo, así como con la Autoridad del Parque, con los municipios afectados con las áreas del párrafo d) del mismo apartado 2, emita la sentencia de su aprobación. Si el plan no es aprobado en menos de veinticuatro meses a partir de la creación del parque, la región se sustituye por una comisión mixta integrada por representantes del Ministerio de Medio Ambiente y representantes de las regiones y provincias autónomas, que hace los esfuerzos necesarios para lograr tales acuerdos, y si los acuerdos en cuestión no se alcanzan dentro de los próximos cuatro meses, el Ministro deberá remitir el asunto al Consejo de Ministros que decide definitivamente.

5. En caso de incumplimiento de las condiciones contempladas en el apartado 3, a la Administración se sustituye el Ministro para el Medio Ambiente, que procede en los mismos términos con un comisario ad acta.

6. El plan se modifica de la misma forma requerida para su aprobación y se actualiza con la misma modalidad por lo menos cada diez años.

7. El plan entra en vigor una declaración de interés público y urgencia e indiferibilidad para los interventos y sustituye a todo nivel los planes de paisaje, los planes urbanos y espaciales o cualquier otra herramienta de planificación.

8. El plan se publicará en la Gaceta Oficial de la República Italiana y en el Boletín Oficial de la región y es inmediatamente vinculante para el gobierno y el sector privado". Parlamento Italiano, Ley 394 de 1991, "Por la cual se establece protección del derecho ambiental frente a la propiedad" (Roma: Gaceta Oficial de la República Italiana y Boletín Oficial, 6 de diciembre de 1991), art. 12. 
alteración del ambiente por actuación del hombre; en las segundas no se permite la construcción o la ampliación de nuevas obras, pero sí la utilización productiva y la construcción de infraestructuras; en las terceras se admiten las actividades del sector primario y artesanal y en las cuartas, las actividades para el desarrollo social y cultural. ${ }^{13}$ Podemos decir que hay zonas en donde prevalece la protección integral de la naturaleza, donde el derecho de propiedad se convierte en un mero título de propiedad y el derecho de goce de la cosa es casi inexistente. En otras zonas, en cambio, el desarrollo económico es visto como útil a las exigencias del parque natural y las exigencias de protección del ambiente también restringen la utilización del bien y en las últimas todavía se permite un uso muy eficiente.

Un efecto parecido tienen los deberes paisajísticos (introducidos por el Código de los bienes culturales y del paisaje ${ }^{14}$ y los deberes urbanísticos-territoriales con finalidad de protección ambiental y paisajística (regulados por la Ley 1150 de 1942). ${ }^{15}$

\section{Limitaciones generales a la propiedad: las inmisiones industriales y sus límites}

\subsection{Las inmisiones y las inmisiones industriales en el Código Civil}

En el párrafo anterior examinamos cómo el Derecho Administrativo, por medio de la remisión que hace el Código Civil, puede establecer límites, a veces muy altos, sobre el goce del bien por parte del propietario con la finalidad de proteger el ambiente. En las próximas líneas veremos cómo estas mismas exigencias pueden poner un "límite general a una excepción a un límite general" de la propiedad. En palabras más claras, revisaremos si la protección del ambiente puede impedir a una industria superar la "tolerabilidad normal" de las inmisiones al fundo vecino, como sería consagrado por el Código Civil en derogación al límite general de las inmisiones. Veremos, en sustancia, si el ambiente puede ser protegido no solo con los instrumentos del Derecho público, sino también con los del Derecho privado: ${ }^{16}$ si el Código italiano en vigor sigue siendo el de 1942, centrado en las relaciones

13 Ciocia, Aree protette e diritto di proprietà, 103-110.

14 Renna, "Vincoli alla proprietà e diritto dell'ambiente", 7.

15 Renna, "Vincoli alla proprietà e diritto dell'ambiente", 8-10. Sobre el tema de los vínculos urbanísticos por las zonas con vocación agrícola y de su finalización a los fines de tutela ambiental, puede verse Angelo Saturno, "Edificazione in zona agricola, valorizzazione del territorio e problematiche in tema di diritto di proprietà", http://www.comparazionedirittocivile.it/prova/files/saturno_edificazione.pdf (acceso febrero 15, 2014).

16 Ugo Salanitro, "Tutela dell'ambiente e strumenti di diritto privato", http://www.lex.unict.it/didattica/materiale08/dirprivatoambiente/lezioni/relazioneprivatenforcement.pdf (acceso febrero 7, 2014). 
económicas, su relación con la Constitución republicana del 1948 no puede no llegar a una reinterpretación de la disciplina en una perspectiva personalista, de modo que sustituya el trabajo en esta dirección que en otros países (como Brasil) logró el legislador.

Como se sabe, la disciplina de las inmisiones constituye la principal limitación al derecho de propiedad, entendido como absoluto. Esto significa que la regla general es la facultad de utilizar y disfrutar del fundo de una manera absoluta, pero el Estado liberal, por medio legislativo o reglamentario, puede establecer limitaciones, para asegurar la paz y la seguridad individual. ${ }^{17}$ En este sentido, la limitación al derecho de propiedad existe solo porque también existe el derecho de propiedad del vecino. ${ }^{18}$ Nos encontramos todavía en la lógica de las relaciones propietarias, no en la lógica de las relaciones interpersonales.

Además, la regla de las limitaciones a la propiedad "como está consagrada en el numeral 1 del Artículo 844 del Código Civil italiano" establece una regulación determinada por el uso normal de la propiedad. En la disciplina específica establecida para los límites de la propiedad de tipo industrial, en el numeral 2 del mismo Artículo, las exigencias productivas tienen más importancia que la propiedad misma. ${ }^{19}$ En este sentido, la propiedad tiene una función orientada a las exigencias de la producción nacional y de la industrialización del país. ${ }^{20}$

Es por eso que la disciplina es parcialmente diferente. Si las inmisiones del numeral 1 son ilícitas cuando sobrepasan la "tolerabilidad normal", ${ }^{21}$ las inmisiones del numeral 2, también en el caso de que la superen, pueden ser consideras lícitas si existen y son prevalentes de las "exigencias de la producción". ${ }^{22}$ En opinión de

Cesare Salvi, Le immissioni industriali: rapporti di vicinato e tutela dell'ambiente (Milán: Giuffrè, 1979), 26.

Salvi, Le immissioni industriali, 32.

Salvi, Le immissioni industriali, 135.

Salvi, Le immissioni industriali, 153. "El propietario no puede evitar la emisión de humo o de calor, humos, ruido, agitaciones y propagaciones similares que resultan de la parte inferior de cierre, si no exceden los niveles normales, también teniendo en cuenta la condición del lugar.

En la aplicación de esta norma, el tribunal debe equilibrar las necesidades de la producción con las razones de la propiedad. Se podrá tener en cuenta la prioridad de un uso específico". Parlamento Italiano, Código Civil (Roma: Pubblicato nella edizione straordinaria della Gazzetta Ufficiale, 1942), art. 844.

21 Puede decirse que cuanto más degradado sea el ambiente, mayor será el nivel de tolerabilidad requerido. Paolo Guarda, "Immissioni: tutela proprietaria e diritto dell'ambiente", http://eprints.biblio.unitn.it/1423/1/ Immissioni_Guarda.pdf (acceso febrero 5, 2012).

22 Salvi, Le immissioni industriali, 201. De una opinión diferente es Giusti, para quien "para las limitaciones generadas por actividades productivas [... ], la utilidad social debe inspirar la valoración de la intolerabilidad". Alberto Giusti, "La proprietà" en Diritto Privato: Parte Seconda (Turín: UTET, 2004), 812. 
Procida Mirabelli di Lauro, ${ }^{23}$ por lo contrario, el límite de la "tolerabilidad normal" permanece también en el caso del numeral 2, pero considerada de una manera más amplia. Desde este entendimiento, muchas sentencias permitieron inmisiones superiores a la "tolerabilidad normal", al asegurar más bien una indemnización equitativa para los propietarios que tuvieron daños derivados de la actividad industrial, en lugar del resarcimiento del daño causado.

La indemnización no es la misma que hay cuando se establece en virtud de una medida coactiva, porque en este caso no es la sentencia lo que legitima las inmisiones, sino que son lícitas desde antes de la decisión del juez, ${ }^{24}$ y es más parecida a las indemnizaciones establecidas en el Derecho Administrativo para las limitaciones sustancialmente expropiatorias de la propiedad privada. ${ }^{25}$ No hay la posibilidad de que el juez imponga la eliminación de las inmisiones si los medios técnicos necesarios no son razonables económicamente ${ }^{26}$ lo que, como veremos, es muy diferente a lo que sucede en la más moderna disciplina de la reparación del daño ambiental.

\subsection{Las limitaciones generales a la propiedad en la Constitución}

En la Constitución republicana, la perspectiva es completamente nueva. Si la propiedad se subordina a la "función social", por la libertad de iniciativa económica, la limitación es doble: por un lado, "no se puede desarrollar contra la utilidad social o perjudicando la seguridad, la libertad y la dignidad humana", ${ }^{27}$ de otro lado, la ley coordinaría la actividad económica para éxitos sociales. ${ }^{28}$

Así, si en la perspectiva del Código Civil de 1942 el derecho más importante del Estado liberal "la propiedad" es limitado por las exigencias productivas, en la perspectiva constitucional las exigencias productivas tienen que ser limitadas por los principios fundamentales, en los cuales comprendemos, como lo señala de modo explícito el Artículo 41, "la seguridad, la libertad y la dignidad humana". No podemos tampoco olvidar otros principios fundamentales explícitos e implícitos en la Constitución, incluida aquella de protección del "paisaje" enunciada por el Artículo 9, numeral

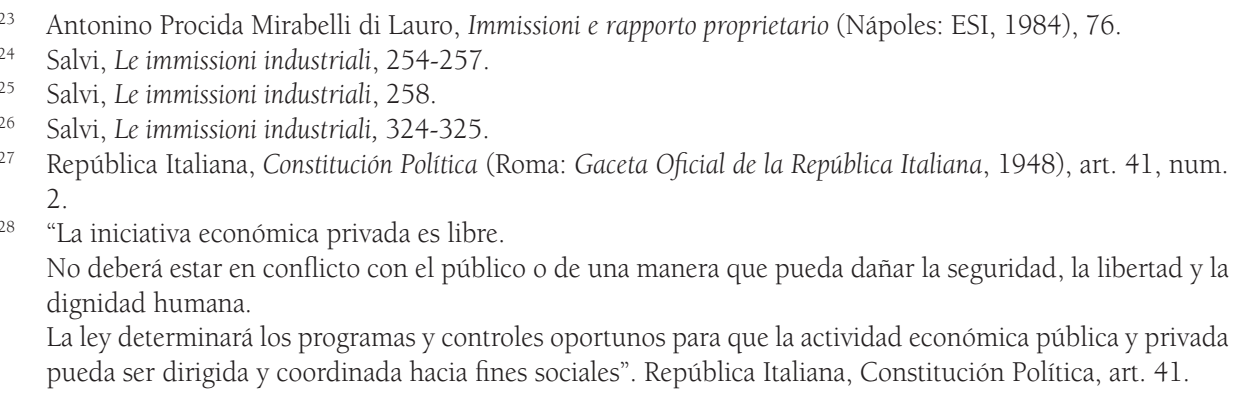


2 , que siempre fue interpretada extensivamente como protección del ambiente, ${ }^{29}$ es decir, el hábitat natural del ser humano y de los ecosistemas ${ }^{30}$ y como derecho a la salubridad del mismo, en conexión con el Artículo 32 de la Constitución, que protege la salud como "derecho fundamental del individuo e interés de la colectividad”, en especial después de que, con el Acto Único Europeo, la protección del ambiente fuera incluida entre los fines de la acción de la Comunidad. Así figura en el Título XX, Artículos 191, ${ }^{31} 192^{32}$ y $193^{33}$ del Tratado sobre el Funcionamiento de la Unión Europea. ${ }^{34}$

29 Sobre la evolución de la jurisprudencia constitucional en la elaboración de un derecho al ambiente, a partir del Artículo 9 y de los Artículos 32 y 44, puede verse Lorenzo Nannipieri, "Il ruolo della Corte nella definizione della materia ambientale", http://www.gruppodipisa.it/wp-content/uploads/2011/07/Nannipieri. pdf (acceso febrero 5, 2014).

30 Jörg Luther, "I principi fondamentali della cultura" en I principi fondamentali della Costituzione italiana (Pisa: Plus, 2002), 130.

31 "1. La política de la Unión en el ámbito del medio ambiente contribuirá a alcanzar los siguientes objetivos:

- la conservación, la protección y la mejora de la calidad del medio ambiente,

- la protección de la salud de las personas,

- la utilización prudente y racional de los recursos naturales,

- el fomento de medidas a escala internacional destinadas a hacer frente a los problemas regionales o mundiales del medio ambiente y en particular a luchar contra el cambio climático.

2. La política de la Unión en el ámbito del medio ambiente tendrá como objetivo alcanzar un nivel de protección elevado, teniendo presente la diversidad de situaciones existentes en las distintas regiones de la Unión. Se basará en los principios de cautela y de acción preventiva, en el principio de corrección de los atentados al medio ambiente, preferentemente en la fuente misma, y en el principio de quien contamina paga.

En este contexto, las medidas de armonización necesarias para responder a exigencias de la protección del medio ambiente incluirán, en los casos apropiados, una cláusula de salvaguardia que autorice a los Estados miembros a adoptar, por motivos medioambientales no económicos, medidas provisionales sometidas a un procedimiento de control de la Unión.

3. En la elaboración de su política en el área del medio ambiente, la Unión tendrá en cuenta:

- los datos científicos y técnicos disponibles,

- las condiciones del medio ambiente en las diversas regiones de la Unión,

- las ventajas y las cargas que puedan resultar de la acción o de la falta de acción,

- el desarrollo económico y social de la Unión en su conjunto y el desarrollo equilibrado de sus regiones. 4. En el marco de sus respectivas competencias, la Unión y los Estados miembros cooperarán con los terceros países y las organizaciones internacionales competentes. Las modalidades de la cooperación de la Unión podrán ser objeto de acuerdos entre ésta y las terceras partes interesadas.

El párrafo precedente se entenderá sin perjuicio de la competencia de los Estados miembros para negociar en las instituciones internacionales y para concluir acuerdos internacionales". Unión Europea, Tratado sobre el funcionamiento de la Unión Europea (Lisboa: Diario Oficial Unión Europea, 26 de octubre, 2012), art. 191.

32 "1. El Parlamento Europeo y el Consejo, con arreglo al procedimiento legislativo ordinario y previa consulta al Comité Económico y Social y al Comité de las Regiones, decidirán las acciones que deba emprender la Unión para la realización de los objetivos fijados en el artículo 191.

2. No obstante el procedimiento de toma de decisiones contemplado en el apartado $1, y$ sin perjuicio del artículo 114, el Consejo, por unanimidad con arreglo a un procedimiento legislativo especial, a propuesta de la Comisión y previa consulta al Parlamento Europeo, al Comité Económico y Social y al Comité de las Regiones, adoptará:

a) disposiciones esencialmente de carácter fiscal;

b) las medidas que afecten a:

- la ordenación territorial;

- la gestión cuantitativa de los recursos hídricos o que afecten directa o indirectamente a la disponibilidad de dichos recursos;

- la utilización del suelo, con excepción de la gestión de los residuos; 
Desde esta perspectiva, no tenemos ninguna regla que defina límites específicos al derecho de propiedad, sino más bien un principio general de protección del ambiente, que constituye una "originaria limitación de la propiedad privada, directamente proporcional a la extensión y a la importancia reconocida al valor ambiental" 35 , importancia que, a su vez, puede cambiar y, con ella, puede cambiar la limitación puesta en general al derecho de propiedad. ${ }^{36}$

c) las medidas que afecten de forma significativa a la elección por un Estado miembro entre diferentes fuentes de energía y a la estructura general de su abastecimiento energético.

El Consejo, por unanimidad, a propuesta de la Comisión y previa consulta al Parlamento Europeo, al Comité Económico y Social y al Comité de las Regiones, podrá disponer que el procedimiento legislativo ordinario sea aplicable a los ámbitos mencionados en el párrafo primero.

3. El Parlamento Europeo y el Consejo adoptarán, con arreglo al procedimiento legislativo ordinario y previa consulta al Comité Económico y Social y al Comité de las Regiones, programas de acción de carácter general que fijen los objetivos prioritarios que hayan de alcanzarse.

Las medidas necesarias para la ejecución de dichos programas se adoptarán de conformidad con las condiciones contempladas en el apartado 1 o en el apartado 2, según proceda.

4. Sin perjuicio de determinadas medidas adoptadas por la Unión, los Estados miembros tendrán a su cargo la financiación y la ejecución de la política en materia de medio ambiente.

5. Sin perjuicio del principio de quien contamina paga, cuando una medida adoptada con arreglo a lo dispuesto en el apartado 1 implique costes que se consideren desproporcionados para las autoridades públicas de un Estado miembro, dicha medida establecerá las disposiciones adecuadas en forma de:

- excepciones de carácter temporal,

- apoyo financiero con cargo al Fondo de Cohesión creado de conformidad con lo dispuesto en el artículo 177, o ambas posibilidades. Unión Europea, Tratado sobre el funcionamiento de la Unión Europea, art. 192.

33 "Las medidas de protección adoptadas en virtud del artículo 192 no serán obstáculo para el mantenimiento y la adopción, por parte de cada Estado miembro, de medidas de mayor protección.

Dichas medidas deberán ser compatibles con los Tratados y se notificarán a la Comisión Unión Europea, Tratado sobre el funcionamiento de la Unión Europea, art. 193.

34 Francesco Martinelli, Diritto dell'Unione Europea: Aspetti istituzionali e politiche dell'Unione 19a ed. (Nápoles: Simone, 2012), 437-453. Muy interesante es la sentencia del Tribunale di Montepulciano, "Ambiente e la tutela della vita”, 27 febrero 2007, n. 46, Rivista giuridica dell'ambiente, núm. 6 (2007): 857-870, que enlaza la protección de las inmisiones con el derecho a la protección de la vida privada y familiar, del domicilio y de la correspondencia, establecido por la Convención Europea de los Derechos del Hombre.

35 Maurizio Renzulli, "Tutele dell'ambiente e proprietà privata", http://www.lexitalia.it/articoli/renzulli_ambiente.htm (acceso febrero 8, 2014).

36 Renzulli, "Tutele dell'ambiente e proprietà privata". Si es claro que la limitación al derecho de propiedad por exigencias ambientales es variable en relación con la época histórica, no parece correcta la tesis de Salvi en cuanto a la imposibilidad de una abstracta jerarquía entre producción industrial, de un lado y salud y ambiente, del otro. En realidad, el Artículo 41, comma 2, de la Constitución dice con claridad que la iniciativa económica privada "no se puede desarrollar contra la utilidad social o perjudicando la seguridad, la libertad y la dignidad humana”. No estamos en frente a dos derechos constitucionales de igual importancia, como parecería a leer a Salvi, sino ante dos derechos constitucionales de los cuales uno (la iniciativa económica privada) está expresamente subordinado a otros (la seguridad, la libertad y la dignidad, entre los que la vida y la salud constituyen inevitable premisa y fines y que se perjudican si el ambiente no es salubre) y, en general, a la utilidad social, que incluye el respeto al ambiente, ya como paisaje ya como salubridad ambiental. Salvi, Le immissioni industriali, 372-373. 


\subsection{Las inmisiones industriales a la luz de la disciplina constitucional de la propiedad en la jurisprudencia}

En la jurisprudencia, después de una primera fase, contemporánea al período de industrialización del país, muy favorable a los intereses de la industria ${ }^{37}$, década del setenta ha ido afirmándose que, en la evaluación del interés del vecino comparado con el del emprendedor, se considera no solo el provecho de este último, sino también el interés al empleo de los trabajadores, como el interés a la producción de bienes. Sin embargo, estos intereses sucumben frente a la protección del ambiente, ${ }^{38}$ y a la indemnización económica se añade el resarcimiento en forma específica, con la restauración del estado de los lugares (desde el Artículo 18, alineado 8, ${ }^{39}$ de la Ley 349 de $1986,{ }^{40}$ ahora abrogado por el Artículo 318, alineado 2, literal a del Decreto Legislativo 152 de 2006), sin la limitación de la excesiva onerosidad. ${ }^{41}$ Esto porque el ambiente es reconocido como bien público (o, más correctamente, como bien común), que pertenece a todos los ciudadanos y, de esta manera, es imposible un resarcimiento del daño equivalente. ${ }^{42}$

Como se ha dicho, en la perspectiva originaria del Código no se requiere limitar las inmisiones si los medios técnicos necesarios para hacerlo son muy costosos; en la jurisprudencia reciente se requiere reducir las inmisiones a un punto que no dañe a los vecinos si las técnicas necesarias son muy onerosas y si son inferiores a lo indicado en las reglas públicas. ${ }^{43}$

3 Salvi, Le immissioni industriali, 348-349.

38 Ugo Mattei, I diritti reali 1: La proprietà (Turín: UTET, 2001), 324-325.

39 "El juez, en la sentencia condenatoria ordena, si es posible, el restablecimiento de las condiciones de los lugares, a expensas de la persona responsable". Parlamento Italiano, Ley 349 de 1986, "Por la cual se da la creación del Ministerio de Medio Ambiente y normas en materia de daño ambiental" (Roma: Gazzetta Ufficiale della Repubblica Italiana, núm. 162, 8 de julio de 1986), art. 18, alineado 8.

40 Sobre los desarrollos de la disciplina, puede verse Franco Giampietro, "La responsabilità per danno all'ambiente in Italia: sintesi di leggi e di giurisprudenza messe a confronto con la direttiva 2004/35/CE e con il T.U. Ambientale", Rivista giuridica dell'ambiente, núm. 1 (2006): 30.

41 Mattei, I diritti reali 1, 325-326.

42 Mattei, I diritti reali 1, 326.

43 Acerca de las inmisiones electromagnéticas, todavía se considera solamente la noción de "tolerabilidad normal" y no se examina el problema desde la perspectiva del comma 2 del Artículo 844, que parece más pertinente al hablar de un electroducto. Tribunale di Como, "Immissione elettromagnetica", 23 noviembre 2005, n. 1490, Rivista giuridica dell'ambiente, núm. 2 (2006): 325-333. 


\subsection{Conseguir mayor protección de la salubridad ambiental por medio de la revisita de la disciplina de las inmisiones}

Respecto a los límites señalados por las leyes y los reglamentos administrativos, para que una inmisión sea lícita, puede no ser suficiente que el vecino que la sufre demuestre que hay un daño. El Artículo 844 solo tutela el derecho de propiedad de los vecinos; si puede haber un efecto de protección del ambiente o de la salud, esto constituye un efecto secundario, que no tiene influencia en la evaluación de la licitud de las inmisiones. ${ }^{44}$

Esta interpretación depende de la idea de que los efectos de una actividad de producción pueden verse en exclusiva en los fundos vecinos. En la moderna sociedad industrial no siempre es así, pues en muchas ocasiones los efectos de esta actividad se reverberan muy lejos. Así, una fábrica puede contaminar regiones enteras, con consecuencias dañinas para la salud de poblaciones de gran tamaño y, en general, para el ambiente, como el reciente caso de la ILVA de Taranto: la más importante industria siderúrgica italiana, que fue intervenida por el Gobierno italiano a causa de los daños al ambiente; es reconocida como una de las fábricas más significativas en Europa en la producción de hierro y cuenta con más de 11.000 trabajadores.

Si, en una perspectiva tradicional, estas emisiones son producidas dentro de los límites legales, tenían que ser toleradas en virtud del numeral 2 del Artículo 844 del Código Civil. En cambio, si la disciplina es vista desde una perspectiva constitucional, esto no es así. En efecto, cuando surgen tensiones entre la propiedad y el derecho a la salud (que incluye el derecho a un ambiente sano), la Constitución protege el segundo; si se demuestra la existencia de un daño real o potencial a la salud, este prevalece, sin que sea relevante el hecho de que el Artículo 844 está comprendido en las reglas puestas a tutela de la propiedad. ${ }^{45}$

Si las emisiones contaminan una región entera, todos los habitantes, en calidad de propietarios, a cuyo cargo es el onus probandi, en mi opinión, pueden alegar las bases del Artículo 84, siempre en los límites legales, para impedir la emisión de sustancias contaminantes y así las inmisiones en sus propios fundos, por medio de una tutela inhibitoria y resarcitoria.

44 Guarda, "Immissioni: tutela proprietaria e diritto dell'ambiente", 15.

45 Guarda, "Immissioni: tutela proprietaria e diritto dell'ambiente", 16. 
La reinterpretación de la disciplina de las inmisiones a la luz de los valores constitucionales está encontrando, aunque tímidamente, el favor de la Corte de Casación. Por ejemplo, en una sentencia de $2006^{46}$ no se otorga relevancia al uso preexistente del fundo de donde vienen las inmisiones, al subrayar que no fueron adoptadas las medidas necesarias para proteger la salud de los vecinos.

\section{Conclusiones}

Ab inferis usque ad sidera: desde hace mucho tiempo esta máxima latina no tiene más validez. En el Código Civil italiano, al lado de algunas limitaciones específicas a las facultades del propietario conexas a minas, tuberías, antigüedades, aguas, obras hidráulicas e hidrocarburos hay una regla general que limita estas facultades. El propietario "no puede impedir las actividades de terceros que se desarrollen a tal profundidad en el subsuelo o a tal altitud en el espacio inminente que él no tenga interés a excluirlos" ${ }^{47}$ En el Código y en las leyes complementarias y administrativas hay muchas limitaciones al goce del bien por parte del propietario, para que no sobrepase los confines del fundo, así como una limitación general de tipo horizontal al goce del fundo, que surge de la disciplina de las inmisiones, que todavía, en la perspectiva originaria, debe evitar que la expansión del derecho de un propietario comporte una compresión del derecho de otro propietario. Aún más, desde la perspectiva constitucional y a la luz de la extensión geográfica de las consecuencias de la actividad industrial, esta misma disciplina puede convertirse en una limitación mucho más amplia: cada propietario puede impedir toda actividad de emisión de sustancias contaminantes que sean dañinas para la salud y para la salubridad ambiental, sin que tenga relevancia el hecho de que se encuentre en los límites legales, que venga de un fundo "vecino" en un sentido estricto y cause daños en fundos lejanos y que las razones de la producción puedan prevalecer.

La jurisprudencia ya se está orientando en dirección de la tutela a la salud. Auspiciamos que pronto serán acogidas por completo las otras consecuencias de una lectura constitucionalmente orientada de la disciplina y que la protección del ambiente venga a ser, de esta manera, un límite general al derecho de propiedad y no solo el fin de las normas específicas que limitan las facultades del propietario. ${ }^{48}$

46 Corte di Cassazione Civile, Sezione III, 11 abril 2006, n. 8420, M. P. Giuseppe Buffone. http://www.altalex. com/index.php?idnot=10507 (acceso febrero 6, 2012).

47 Parlamento Italiano, Código Civil, art. 840, num. 2.

48 Para profundizar en estos temas, puede verse, además, Mariachiara Alberton, "Il danno ambientale in un'ottica multilivello: spunti di riflessione", http://www3.unisi.it/ianus/Numero\%202/01.\%20Mariachiara\%20Alber- 


\section{Referencias}

Alberton, Mariachiara. "Il danno ambientale in un'ottica multilivello: spunti di riflessione". http://www3.unisi.it/ianus/Numero\%202/01.\%20Mariachiara\%20.pdf (acceso febrero $5,2014)$.

Baseggio, Carlo. "La disciplina giuridica delle aree naturali protette, tra tutela dell'ambiente e governo del territorio". http://amsdottorato.cib.unibo.it/121/1/Tesi_Baseggio_Carlo. pdf (acceso agosto 1, 2012).

Caretti, Paolo y Ugo de Siervo. Istituzioni di diritto pubblico. 7a ed. Turín: Giappichelli, 2004.

Ciocia, Maria Antonia. Aree protette e diritto di proprietà: Vincoli urbanistici ed uso del territorio. Padua: Cedam, 1999.

Cordini, Giovanni. "Il danno ambientale e la comunità internazionale". http://www.comune. re.it/retecivica/urp/retecivi.nsf/PESIdDoc/E7E35ADEA749F1FFC125787600303CC 6/\$file/relazione\%20Prof\%20Cordini.pdf (acceso febrero 8, 2014).

Corte di Cassazione Civile. Sezione III, 11 abril 2006, n. 8420. M. P. Giuseppe Buffone. http:// www.altalex.com/index.php?idnot=10507 (acceso febrero 6, 2012).

De Martino, Francesco. Della proprietà. 6a ed. Bolonia: Zanichelli, 1976.

Dini, Veronica. "Il diritto soggettivo all'ambiente". http://www.giuristiambientali.it/ documenti/20041124_AM_BCA.pdf (acceso febrero 2, 2014).

Gambaro, Antonio. Il diritto di proprietà. Milán: Giuffré, 1995.

Gargallo di Castel Lentini, Filippo. "Lambiente come diritto fondamentale dell'uomo". http://www.dirittoambiente.net/file/vari_articoli_170.pdf (acceso febrero 4, 2014).

Garzia, Giuseppe. "Costituzione europea e tutela dell'ambiente: riflessioni e problemi aperti”. http://www.centrostudinatura.it/public2/documenti/860-65219.pdf (acceso febrero 4, 2014).

Gaverini, Fabrizio. "La protezione dell'ambiente fra solidarietà e mercato". http://aisberg. unibg.it/bitstream/10446/91/1/FabrizioGaverini_ProtezioneAmbiente.pdf (acceso febrero 5, 2014).

ton.pdf (acceso febrero 5, 2014); Giovanni Cordini, "Il danno ambientale e la comunità internazionale", http://www.comune.re.it/retecivica/urp/retecivi.nsf/PESIdDoc/E7E35ADEA749F1FFC125787600303C C6/\$file/relazione\%20Prof\%20Cordini.pdf (acceso febrero 8, 2014); Veronica Dini, "Il diritto soggettivo all'ambiente", http://www.giuristiambientali.it/documenti/20041124_AM_BCA.pdf (acceso febrero 2, 2014); Giuseppe Garzia, "Costituzione europea e tutela dell'ambiente: riflessioni e problemi aperti”. http:// www.centrostudinatura.it/public2/documenti/860-65219.pdf (acceso febrero 4, 2014); Fabrizio Gaverini, "La protezione dell'ambiente fra solidarietà e mercato", http://aisberg.unibg.it/bitstream/10446/91/1/ FabrizioGaverini_ProtezioneAmbiente.pdf (acceso febrero 5, 2014); Flora Vollero, "Diritti umani e diritti fondamentali fra tutela costituzionale e tutela sovranazionale: il diritto ad un ambiente salubre", http://files. studiperlapace.it/spp_zfiles/docs/20041205175248.pdf/ (acceso febrero 8, 2014). 
Giampietro, Franco. "La responsabilità per danno all'ambiente in Italia: sintesi di leggi e di giurisprudenza messe a confronto con la direttiva 2004/35/CE e con il T.U. Ambientale". Rivista giuridica dell'ambiente, núm. 1 (2006): 19-35.

Giusti, Alberto. "La proprietà" en Diritto Privato: Parte Seconda, editado por Enrico Gabrielli, 5-23. Turín: UTET, 2004.

Guarda, Paolo. "Immissioni: tutela proprietaria e diritto dell'ambiente". http://eprints.biblio. unitn.it/1423/1/Immissioni_Guarda.pdf (acceso febrero 5, 2014).

Luther, Jörg. "I principi fondamentali della cultura" en I principi fondamentali della Costituzione italiana, compilado por Jörg Luther, Elena Malfatti y Emanuele Rossi, 125-154. Pisa: Plus, 2002.

Martinelli, Francesco. Diritto dell'Unione Europea: Aspetti istituzionali e politiche dell'Unione. 19a ed. Nápoles: Simone, 2012.

Mattei, Ugo. I diritti reali 1: La proprietà. Turín: UTET, 2001.

Nannipieri, Lorenzo. "Il ruolo della Corte nella definizione della materia ambientale". http://www.gruppodipisa.it/wp-content/uploads/2011/07/Nannipieri.pdf (acceso febrero 5, 2014).

Parlamento Italiano. Código Civil. Roma: Pubblicato nella edizione straordinaria della Gazzetta Ufficiale, 1942.

Parlamento Italiano. Ley 349 de 1986, "Por la cual se da la creación del Ministerio de Medio Ambiente y normas en materia de daño ambiental. Roma: Gazzetta Ufficiale della Repubblica Italiana, núm. 162, 8 de julio de 1986.

Parlamento Italiano. Ley 394 de 1991, "Por la cual se establece protección del derecho ambiental frente a la propiedad". Roma: Gaceta Oficial de la República Italiana y Boletín Oficial, 6 de diciembre de 1991.

Procida Mirabelli di Lauro, Antonino. Immissioni e rapporto proprietario. Nápoles: ESI, 1984. Pugliatti, Salvatore. La proprietà nel nuovo diritto. Milano: Giuffrè, 1954.

Renna, Mauro. "Vincoli alla proprietà e diritto dell'ambiente". http://www.astrid-online. it/Gli-osserv/llpp/M_Renna-Vincoli-alla-propriet--08_09_06.pdf (acceso febrero 6, 2014).

Renzulli, Maurizio. "Tutele dell'ambiente e proprietà privata”. http://www.lexitalia.it/articoli/ renzulli_ambiente.htm (acceso febrero 8, 2014).

República Italiana. Constitución Política. Roma: Gaceta Oficial de la República Italiana, 1948. Rodota, Stefano. Il diritto di avere diritti. Roma: Laterza, 2012.

Salanitro, Ugo. "Tutela dell'ambiente e strumenti di diritto privato". http://www.lex.unict. it/didattica/materiale08/dirprivatoambiente/lezioni/relazioneprivatenforcement. pdf (acceso febrero 7, 2014).

Salvi, Cesare. Le immissioni industriali: Rapporti di vicinato e tutela dell'ambiente. Milán: Giuffrè, 1979. 
Saturno, Angelo. "Edificazione in zona agricola, valorizzazione del territorio e problematiche in tema di diritto di proprietà". http://www.comparazionedirittocivile.it/prova/files/ saturno_edificazione.pdf (acceso febrero 15, 2014).

Scarcella, Alessio. "Giurisprudenza C.e.d.u. e diritto dell'ambiente: i principali 'filoni' della Corte di Strasburgo". Ambiente e sviluppo, núm. 2 (2013): 129-137.

Sertorio, Marco. "Attività estrattiva e tutela ambientale". http://www.assomineraria.org/ news/attach/copy_0_sertorio_attivit_estrattiva_e_tutela_ambientale.pdf (acceso febrero 10, 2014).

Torrente, Andrea y Piero Schlesinger. Manuale di diritto privato. 10a ed. Milán: Giuffré, 1978.

Tribunale di Como. "Immissione elettromagnetica". 23 noviembre 2005, n. 1490, Rivista giuridica dell'ambiente, núm. 2 (2006): 325-333.

Tribunale di Montepulciano. "Ambiente e la tutela della vita". 27 febrero 2007, n. 46. Rivista giuridica dell'ambiente, núm. 6 (2007): 857-870.

Unión Europea. Tratado sobre el funcionamiento de la Unión Europea. Lisboa: Diario Oficial Unión Europea, 26 de octubre, 2012.

Vollero, Flora. "Diritti umani e diritti fondamentali fra tutela costituzionale e tutela sovranazionale: il diritto ad un ambiente salubre". http://files.studiperlapace.it/ spp_zfiles/docs/20041205175248.pdf (acceso febrero 8, 2014).

Vollero, Flora. "Il diritto all'ambiente salubre nell'elaborazione della giurisprudenza di Strasburgo". http://www.diritto.it/materiali/ambiente/vollero.html (acceso febrero 5, 2014). 